\title{
Meningitis criptocóccica en pacientes sin infección por VIH: presentación de dos casos y revisión de la literatura
}

\author{
Cristhian Ramírez-Ramos',2, Juan Galindo², Stephany Correa², German Giraldo-Bahamon ",2, \\ Juan Rivera², Juan Solano², María Peralta² y Guillermo González-Manrique 1,2,3
}

'Departamento de Medicina Interna, Universidad Surcolombiana, Hospital Universitario Hernando Moncaleano Perdomo, Neiva, Colombia.

${ }^{2}$ Semillero de investigación en Medicina Interna -SIMI. Grupo de investigación MI-Dneuropsy, Universidad Surcolombiana, Neiva, Colombia.

${ }^{3}$ Neurólogo clínico- internista. Jefe del servicio de neurofisiología. Hospital Universitario Hernando Moncaleano Perdomo Neiva, Colombia.

Recibido: 13 de octubre de 2017 Aceptado: 13 de noviembre de 2018

Correspondencia a: Cristhian Ramírez-Ramos cristhianramos1989@hotmail.com

\section{Introducción}

C ryptococcus spp. es un hongo encapsulado que causa infecciones en los humanos, desde una colonización pulmonar asintomática hasta meningoencefalitis ${ }^{1}$. Afecta principalmente a pacientes inmunocomprometidos (infección por VIH, tratamiento prolongado con glucocorticoides, sometidos a trasplante de órganos, cáncer, sarcoidosis, etc) y con menos frecuencia a individuos sin condiciones de predisposición ${ }^{1,2}$. El compromiso del sistema nervioso central (SNC) y pulmonar son los más frecuentes. La carga global de la infección es alta, en especial en países en vías de desarrollo. Las tasas de mortalidad estimada en Norteamérica varían entre 9-20\%, mientras que en el África Sub-sahariana pueden alcanzar $70 \% 0^{3}$, con más de 600.000 muertes en el mundo ${ }^{4}$.

Entre las numerosas especies de Cryptococcus, sólo algunas pueden infectar a los humanos: Cryptococcus neoformans var grubii y Cryptococcus neoformans var neoformans, los cuales afectan a pacientes inmunocomprometidos y Cryptococcus gattii, que puede causar enfermedad tanto en individuos inmunocompetentes como con compromiso inmunológico ${ }^{4}$. La forma meníngea es la causa más común de meningitis en adultos, en países con altas tasas de infección por $\mathrm{VIH}^{5}$. Sin embargo, en los últimos años se ha presentado un incremento en el número de casos en personas con otras formas de inmunosupresión (tanto primaria como secundaria) y en inmunocompeten- tes ${ }^{6}$. En E.U.A, datos de las hospitalizaciones desde 1997 a 2009 reportan que $21,6 \%$ de los casos se presentaron en personas sin VIH, con una mortalidad mayor comparada con los individuos infectados $(13,3 \%$ vs $10,5 \%$; $\mathrm{p}<0,0001)^{7}$. Lo anterior, junto con la comunicación de brotes epidémicos por $C$. gattii en personas aparentemente sanas $^{8}$, denotan la amenaza potencial que representa este agente patógeno.

Presentamos dos casos clínicos, uno en un paciente sin inmunosupresión, y el otro con una inmunosupresión secundaria a su enfermedad de base.

\section{Casos clínicos}

\section{Caso 1}

Varón de 48 años, conductor de taxi, sin ningún antecedente de importancia. Ingresó por un cuadro clínico de dos meses de evolución de cefalea holocraneana de predominio frontal, 8/10 de intensidad en la escala subjetiva del dolor, que se había exacerbado en la última semana previo a su ingreso, a pesar del manejo analgésico convencional. La cefalea estaba asociada a vómitos intermitentes y alteración de la conducta, como agresividad, desorientación, lenguaje incoherente y desconexión del medio, episodios de alrededor de 30 min de duración, sin movimientos anormales, sin fiebre ni otros síntomas asociados. Al examen físico se constató un temblor de 
intención en las extremidades superiores, marcha con ligera lateropulsión izquierda e hiperreflexia global, presión arterial de 160/100 mm Hg y resto del examen físico normal. Sus exámenes de ingreso evidenciaron una leucocitosis de $13.200 / \mathrm{mm}^{3}$ con $71 \%$ de neutrofilia, PCR de $0,33 \mathrm{mg} / \mathrm{dl}$. El estudio microbiológico inicial fue negativo (incluido serología ELISA para VIH y VDRL) y con TC cerebral normal (Figura 1A). Se realizó una punción lumbar (PL), con salida de LCR incoloro transparente, presión de apertura elevada $\left(52 \mathrm{~cm} \mathrm{H}_{2} \mathrm{O}\right)$ y el citoquímico presentó una hipoglucorraquia (valor inferior a lectura de equipo) y proteinorraquia de $106 \mathrm{mg} / \mathrm{dl}$, con 3 leucocitos/ $\mathrm{mm}^{3}$. La baciloscopia y ADA en LCR fueron negativos. La tinta china, látex y reacción de polimerasa en cadena (RPC) fueron positivas para Cryptococcus, por lo que se diagnosticó una meningitis criptocóccica. En el cultivo de LCR se identificó Cryptococcus neoformans. Se inició manejo con fluconazol (800 mg iv al día, fraccionado cada $12 \mathrm{~h}$ ) y anfotericina $\mathrm{B}$ deoxicolato (50 $\mathrm{mg}$ iv al día- dosis calculada $0,7 \mathrm{mg} / \mathrm{kg} /$ día) por cuatro semanas para la fase de inducción.

El paciente persistió con cefalea moderada, vómitos ocasionales y presión arterial elevada, por lo que se realizaron PL diarias para controlar los signos de hipertensión endocraneana (HEC). Se descartó una inmunosupresión secundaria (hepatopatía, enfermedad renal o neumopatía crónica) y se realizó recuento de inmunoglobulinas, subpoblaciones linfocitarias y complemento, todos normales. Por persistencia de signos de HEC se practicó una derivación ventriculoperitoneal. Tres días después del procedimiento el paciente presentó fiebre y rigidez de nuca. Se realizó una nueva PL que mostró persistencia de la hipoglucorraquia e hiperproteinorraquia, con lactato alto en LCR, por lo que se consideró una meningitis bacteriana nosocomial. Se agregaron antimicrobianos de amplio espectro (vancomicina $1 \mathrm{~g}$ y meropenem $2 \mathrm{~g}$ iv por dosis, ambos fraccionados cada $8 \mathrm{~h}$ ). El paciente permaneció con fiebre y rigidez de nuca, por lo que se retiró el catéter de derivación y se retomaron las PL evacuadoras diarias, ante la sospecha de un inóculo persistente. Todos los cultivos fueron negativos. Pese al manejo, presentó deterioro neurológico progresivo, requiriendo soporte ventilatorio y traslado a unidad de cuidado intensivo (UCI). Se tomó TC cerebral de control (Figura 1B) que mostró una hidrocefalia supra-tentorial, por lo que se se planteó realizar un ventriculostomía externa de urgencia. En pabellón quirúrgico se pesquisó una midriasis bilateral plena sin reacción a la luz, sin reflejos de tronco cerebral, confirmando posteriormente una muerte encefálica.

\section{Caso clínico 2}

Mujer de 67 años, dueña de casa, consultó por una semana de evolución de deterioro de su estado general con astenia, adinamia, postración, somnolencia y lenguaje incoherente; sin fiebre, vómitos u otros síntomas asociados. Tenía antecedentes de hipertensión arterial, enfermedad de nodo sinusal usuaria de marcapasos, fibrilación auricular paroxística y cirrosis hepática de etiología no clara en estadio Child Pugh B. Recibía rivaroxaban, espironolactona, propranolol y pantoprazol. Ingresó en regular estado

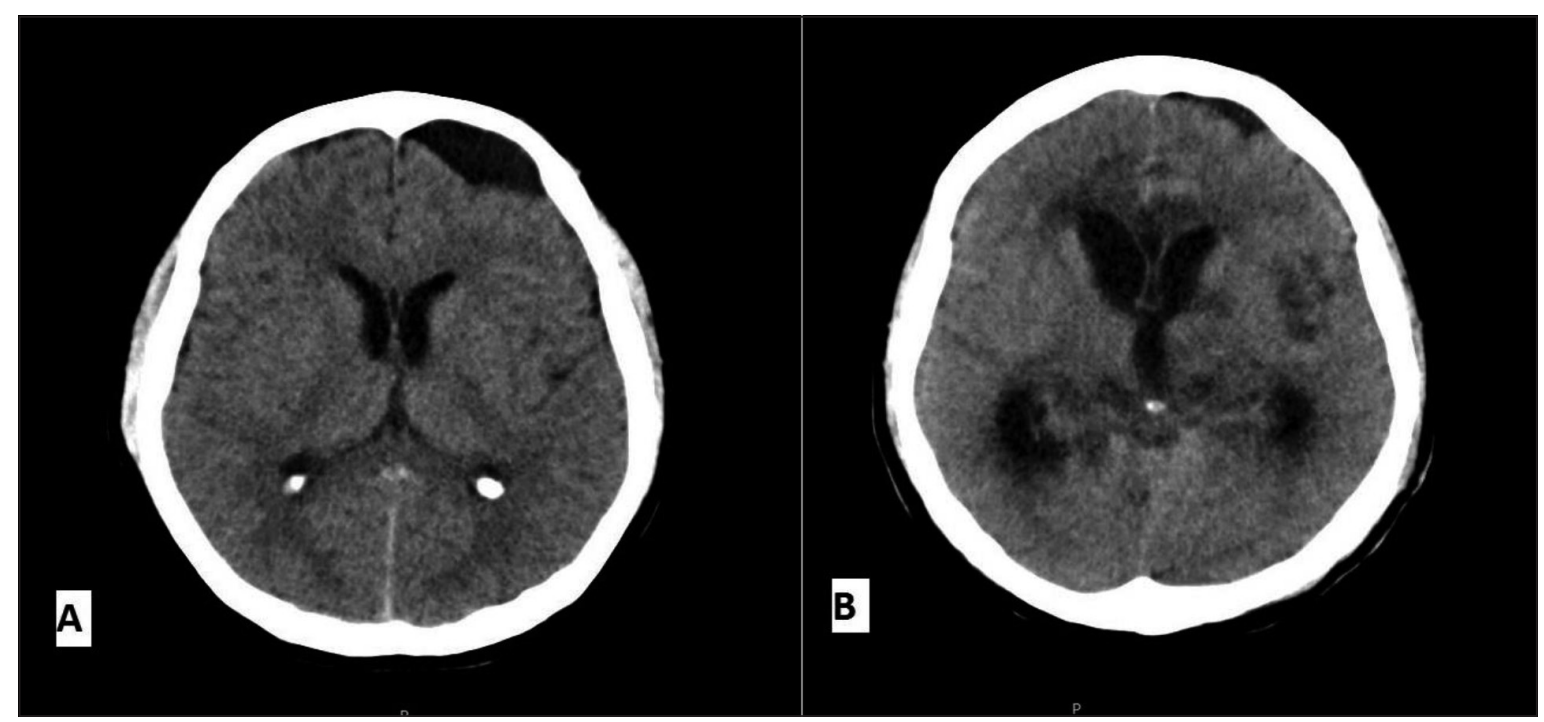

Figura 1. Tomografía de cráneo simple. Cortes axiales. A. Al ingreso: Quiste aracnoideo frontal izquierdo, con tejido cerebral sin lesiones ocupantes de espacio, sin áreas de atenuación o realce anormal que sugieran eventos isquémicos o hemorrágicos, de naturaleza tanto antigua o reciente. Ventrículos de configuración usual. B. Tres días después de retirar derivación ventricular. Importante dilatación de cuernos anteriores y posteriores de los ventrículos laterales, así como dilatación del tercer ventrículo. Hay hipodensidades difusas de predominio periventriculares y parietal izquierdo. 


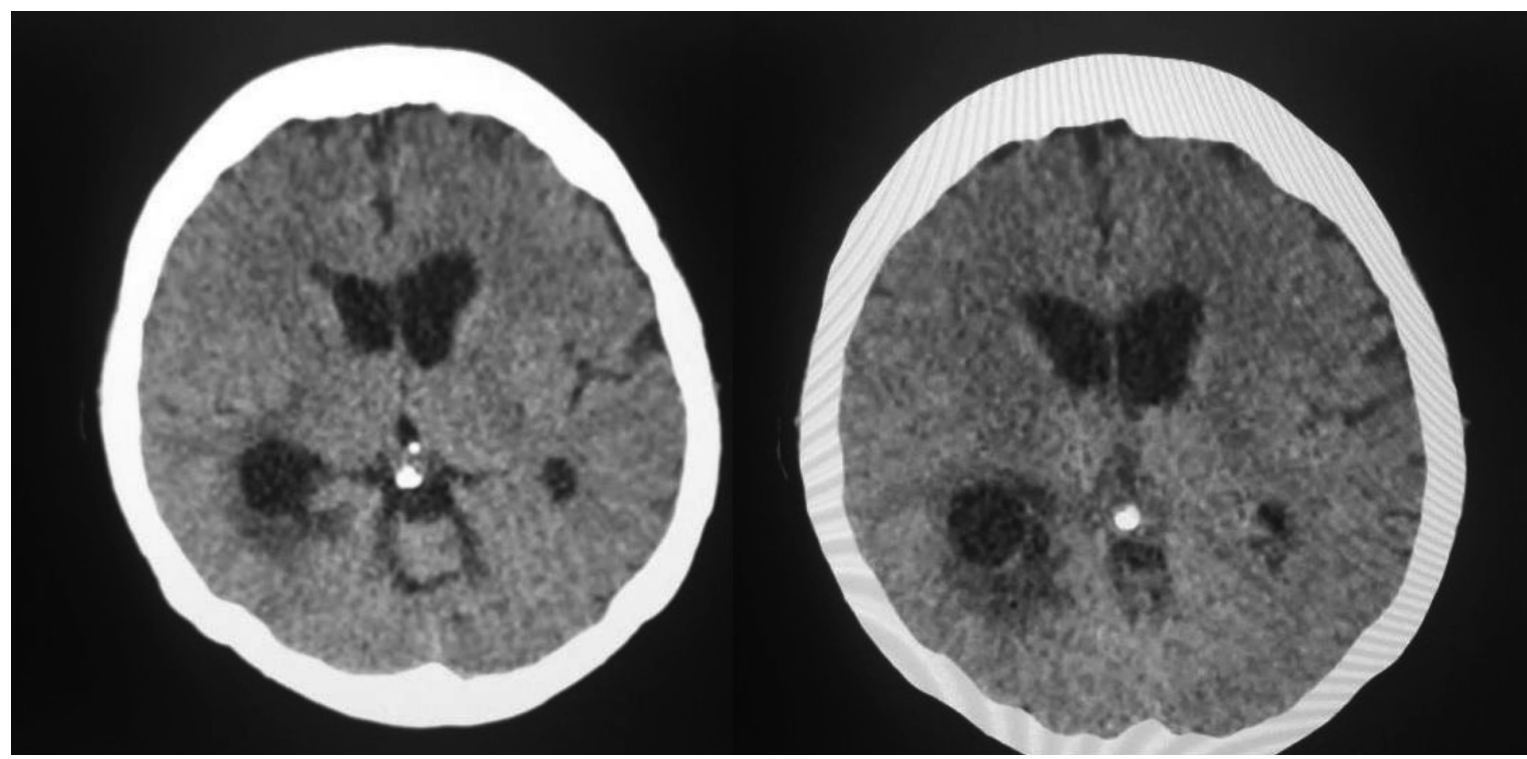

Figura 2. Tomografía de cráneo simple. Cortes axiales. Lesión hipodensa regular que compromete la sustancia blanca profunda adyacente al atrio del ventrículo lateral derecho hasta la porción temporal de este ventrículo lateral con desplazamiento del mismo. Edema vasogénico perilesional.

general, con signos vitales normales. Al examen neurológico presentaba somnolencia, con hemiparesia izquierda, sin asterixis, ni otros hallazgos. Los exámenes tomados al ingreso mostraron un hemograma con 5.050 leucocitos/ $\mathrm{mm}^{3}, 88 \%$ de neutrófilos, trombocitopenia de 61.000/ $\mathrm{mm}^{3}$ e hiponatremia de $122 \mathrm{meq} / 1$, sin otras alteraciones. La TC cerebral evidenció una lesión hipodensa a nivel temporo-occipital derecha, con edema vasogénico (Figura 2). Inicialmente se sospechó una encefalopatía de origen metabólico, en relación con el trastorno electrolítico; sin embargo, no hubo mejoría de los síntomas tras la corrección de las anormalidades metabólicas. Ante los hallazgos de la neuroimagen, se decidió descartar una infección del SNC (meningitis vs absceso cerebral). Se realizó una PL con salida de LCR amarillento, levemente turbio, con un leve aumento de la presión de apertura $\left(22 \mathrm{~cm} \mathrm{H}_{2} \mathrm{O}\right)$. El análisis citoquímico de LCR demostró una pleocitosis linfocítica (100 leucocitos $\left./ \mathrm{mm}^{3}\right)$, hipoglucorraquia (11 mg/dl), hiperproteinorraquia (138 mg/dl), con tinción de tinta china, aglutinación de látex de LCR reactiva para Cryptococcus y cultivo de LCR positivo para C. neorformans. Inició fluconazol $800 \mathrm{mg}$ iv al día, fraccionado cada $12 \mathrm{~h}$ y anfotericina B deoxicolato $60 \mathrm{mg}$ iv al día (dosis calculada $0,7 \mathrm{mg} / \mathrm{kg} /$ día) como tratamiento de inducción de cuatro semanas. Se descartó una infección por VIH con serología ELISA negativa. Se planteó inicialmente un estudio con RM cerebral y derivación para manejo de una hidrocefalia no comunicante; sin embargo, debido a co-morbilidades (dispositivo cardíaco- marcapasos) y trombocitopenia, estos procedimientos se pospusieron. $\mathrm{Su}$ evolución neurológica fue tórpida, presentando compromiso persistente del estado de alerta y además aumento del trabajo respiratorio, con desaturación y evidencia radiográfica de neumonía multilobar, condicionando una falla ventilatoria. Evolucionó con una disfunción orgánica múltiple y falleció en las horas siguientes a su ingreso a UCI. Por la rápida evolución no fue posible realizar estudios para descartar otras alteraciones inmunológicas.

\section{Discusión}

Presentamos dos casos de meningitis criptocóccica en pacientes sin infección por VIH; uno asociado a una patología reconocida por generar disfunción del sistema inmune (cirrosis hepática) con un curso clínico inusual, manifestándose con alteración del sensorio asociado a focalización neurológica y otro en un individuo inmunocompetente quien debutó con una cefalea subaguda y en que se descartó una anormalidad inmunológica de base o alguna condición de riesgo para presentar la infección.

La enfermedad criptocóccica o criptococosis se refiere a la infección causada por hongos del género Cryptococcus. Su incidencia aumentó de manera dramática con el inicio de la epidemia del $\mathrm{VIH}^{9}$ y actualmente ha adquirido mayor significancia en personas receptoras de trasplante de órganos ${ }^{10} y$ con defectos en la inmunidad celular ${ }^{7}$. En una serie clínica de 306 pacientes con criptococosis sin VIH, la mitad presentó compromiso del SNC; de estos $25 \%$ consumía corticoesteroides, $24 \%$ tenían enfermedad pulmonar, renal o hepática crónica, 16\% tenía una 
malignidad y $15 \%$ habían sido sometidos a trasplante de órganos sólidos ${ }^{1}$. Las neoplasias hematopoyéticas tienen un riesgo mayor de presentar esta infección ${ }^{1,2}$. También, se ha descrito la asociación de criptococosis con sarcoidosis, y enfermedades autoinmunes como lupus eritematoso sistémico, hepatitis autoinmune, dermatomiositis y espondilitis anquilosante.

Esta enfermedad en individuos "previamente sanos" es relativamente infrecuente, con aproximadamente 3.000 casos al año en E.U.A., y una incidencia de un caso por 100.000 individuos/año ${ }^{11}$; no obstante, podría corresponder a personas que portan defectos inmunes primarios inusuales. La linfopenia idiopática de células CD4 es la inmunodeficiencia más común relacionada a esta patología, siendo la criptococosis la infección más frecuente en casi un tercio de los casos ${ }^{12}$. La proteinosis alveolar pulmonar (con auto-anticuerpos contra el factor estimulante de colonias granulocito macrófago [FECGM]) también se ha relacionado a infecciones intracelulares, incluida la criptococosis ${ }^{13}$. Algunos individuos aparentemente inmunocompetentes, portadores de esta enfermedad, presentan anticuerpos contra el factor de crecimiento (FEC-GM), lo que genera la inhibición de la señalización a nivel del macrófago, vía fosforilación de $\mathrm{STAT}^{14}$. Otros síndromes monogénicos asociados con criptococosis son la monocitopenia idiopática esporádica de transmisión autosómica dominante por mutaciones en el factor de transcripción GATA2, la inmunodeficiencia hiper-IgM ligada al cromosoma $\mathrm{X}$ y el síndrome de hiperinmunoglobulina E.

El microorganismo se adquiere por la inhalación de esporas y se disemina luego de un período latente, desde los ganglios linfáticos del pulmón. Este patógeno tiene una predilección por el SNC, vinculado a varios factores de virulencia que le permiten penetrar la barrera hematoencefálica, entre ellos metaloproteinasas y ureasas específicas. El compromiso del SNC en la criptococosis se presenta como una meningoencefalitis sub-aguda.

Los síntomas más comunes de la meningitis criptocócica son la cefalea y la alteración del estado mental. El tiempo entre el inicio de los síntomas y la presentación de la meningitis es más prolongado, hasta seis a doce semanas, en personas sin VIH. Los síntomas visuales, como la diplopia y disminución de la agudeza visual, son frecuentes y tardíos en el curso de la enfermedad. Sin tratamiento, la enfermedad progresa y aparecen los estados de confusión, convulsiones, alteración de la conciencia y coma.

Las personas sin historia de VIH muestran una importante variedad de presentaciones clínicas influenciadas no sólo por la respuesta inmune del huésped, sino también por la especie del agente. Los pacientes sometidos a trasplantes de órganos sólidos, de médula ósea o en quimioterapia pueden tener cursos clínicos más cortos, con una respuesta inflamatoria limitada o bien presentarse con características similares a un síndrome de reconstitución inmune ${ }^{15}$. Los pacientes sin VIH infectados con C. neoformans o C. gattii, típicamente tienen un curso clínico larvado de la infección meníngea, a menudo sin fiebre. Las secuelas inflamatorias, como la hidrocefalia, pueden estar presentes al momento del diagnóstico o durante el tratamiento y se ha descrito con mayor frecuencia con C. gattii ${ }^{16}$, probablemente por la mayor producción de citoquinas pro-inflamatorias generada por este agente ${ }^{17}$. En quienes no ocurre la reconstitución inmune, el deterioro clínico se puede presentar durante el tratamiento, no por una falla microbiológica sino por una respuesta inflamatoria post-infecciosa. Las diferencias primarias entre el escenario con o sin infección por VIH incluyen la compartimentalización, con sólo una respuesta intratecal medible y en muchos casos con una activación de macrófagos infiltrantes diferente que generalmente no es protectora, según se ha documentado en modelos animales ${ }^{18}$. El síndrome de respuesta inflamatoria post-infecciosa comparte características con el de reconstitución inmunológica que incluyen: activación de la interacción células dendríticas -células $\mathrm{T}$ y respuesta incrementada de las células $\mathrm{T}$ con niveles altos de interferón gamma e interleuquina- 6; no obstante, difieren en la carencia de activación efectiva de macrófagos, lo que genera una disociación células T- macrófagos, que se traduce en la inflamación y antígenos tisulares persistentes.

El diagnóstico de la infección se basa en el estudio del líquido LCR: generalmente se encuentran presiones de apertura elevadas, con una pleocitosis linfocítica, proteínas elevadas y niveles de glucosa bajos. Las pruebas de aglutinación en látex y el cultivo tienen un buen rendimiento diagnóstico, aunque estos pueden ser negativos, en especial con $C$. gattii. De manera interesante, se han desarrollado las pruebas de flujo lateral que tienen una sensibilidad 100 veces mayor para los polisacáridos de cuatro serotipos de Cryptococcus, comparado con las pruebas de aglutinación en látex ${ }^{19}$.

El tratamiento antimicrobiano se basa en tres etapas: inducción, consolidación y mantención. Las guías de la Sociedad Americana de Enfermedades Infecciosas identifican tres categorías de riesgo para el tratamiento: individuos con infección por VIH, receptores de trasplante de órganos y pacientes sin $\mathrm{VIH}$ - sin trasplantes ${ }^{20}$; aunque los fármacos son los mismos, la duración de la terapia, la dosis y la formulación antifúngica varía dependiendo de la categoría de riesgo. La asociación de anfotericina $\mathrm{B}$ y flucitosina ha demostrado una resolución más rápida de la infección ${ }^{21,22}$ y una mayor supervivencia comparado con anfotericina como monoterapia. La reducción fue de casi $40 \%$ en el riesgo relativo de muerte a las 10 semanas con la adición de flucitosina ${ }^{23}$. En los pacientes sin VIH, la respuesta clínica depende del control de la respuesta inmune y de la infección inicial. El tratamiento con anfotericina 
Tabla 1. Tratamientos antimicrobianos recomendados en criptococosis meníngea en pacientes sin VIH

\begin{tabular}{|c|c|c|}
\hline Estadio & Pacientes trasplantados & Todos los otros pacientes \\
\hline Inducción & $\begin{array}{l}\text { Anfotericina B liposomal 3-6 mg/kg día o anfotericina B } \\
\text { deoxicolato 0,7-1 mg/kg (preferida presentación liposomal } \\
\text { en este grupo) con flucitosina } 100 \mathrm{mg} / \mathrm{kg} \text { día } \\
\text { Duración mínima } 2 \text { semanas }\end{array}$ & $\begin{array}{l}\text { Anfotericina B liposomal 3-6 mg/kg día o anfotericina B } \\
\text { deoxicolato 0,7-1 mg/kg (preferida presentación liposomal } \\
\text { en este grupo) con flucitosina } 100 \mathrm{mg} / \mathrm{kg} \text { día } \\
\text { Duración 4-6 semanas }\end{array}$ \\
\hline Consolidación & $\begin{array}{l}\text { Fluconazol } 400-800 \text { mg día } \\
\text { Duración } 8 \text { semanas }\end{array}$ & $\begin{array}{l}\text { Fluconazol } 400-800 \text { mg día } \\
\text { Duración } 8 \text { semanas }\end{array}$ \\
\hline Mantención & $\begin{array}{l}\text { Fluconazol } 200 \text { mg día } \\
\text { Duración } 6 \text { meses a } 1 \text { año }\end{array}$ & $\begin{array}{l}\text { Fluconazol } 200 \text { mg día } \\
\text { Duración } 6 \text { meses a } 1 \text { año }\end{array}$ \\
\hline $\begin{array}{l}\text { Sin disponibilidad de flucitosina (como en } \\
\text { Colombia) }\end{array}$ & $\begin{array}{l}\text { Adicionar fluconazol 800-1.200 mg día } \\
\text { Duración } 2 \text { semanas }\end{array}$ & $\begin{array}{l}\text { Adicionar fluconazol 800-1.200 mg día } \\
\text { Duración 4-6 semanas }\end{array}$ \\
\hline
\end{tabular}

B asociado a flucitosina es similar al de los pacientes con $\mathrm{VIH}$, pero la fase de inducción debe ser mayor (cuatro a seis semanas) en individuos sin VIH no trasplantados ${ }^{24}$. En escenarios de recursos limitados, la terapia se basa en la disponibilidad de los agentes antifúngicos: fluconazol en dosis altas con flucitosina o con anfotericina B deoxicolato o bien monoterapia con anfotericina B. Anfotericina B es preferida como terapia de inducción comparado con fluconazol. En un estudio de eficacia entre anfotericina B y flucitosina vs. anfotericina B y fluconazol no se encontraron diferencias en cuanto a la tasa de aclaramiento de las ufc de Cryptococcus en LCR. En el primer grupo, 21 pacientes fueron tratados con anfotericina $\mathrm{B}$ a dosis de $0,7-1 \mathrm{mg} / \mathrm{kg} /$ día más flucitosina $25 \mathrm{mg} / \mathrm{kg}$, cuatro veces al día y el segundo grupo incluyó 22 pacientes con anfotericina B, en iguales dosis, y fluconazol, $800 \mathrm{mg}$ al día $^{25}$. Un meta-análisis demostró que a los tres meses no hubo diferencias en mortalidad en estos regímenes de tratamiento, con una tasa de eventos adversos similares ${ }^{26}$. La combinación fluconazol y flucitosina fue superior a fluconazol en el aclaramiento del $\mathrm{LCR}^{20}$. En nuestros pacientes se utilizó la combinación de fluconazol con anfotericina B por no disponer de flucitosina. En la Tabla 1 se muestran los esquemas de tratamiento recomendados.

Los pacientes con linfopenia idiopática de CD4 pueden tener una mayor demora en la respuesta microbiológica, aunque generalmente evolucionan sin secuelas, en contraste con los pacientes con recuento normal de LT CD4. La reducción de la inmunosupresión en pacientes sometidos a trasplante de órganos sólidos es una aproximación lógica después de una infección grave como una criptococosis; aunque la descontinuación de los agentes anti-calcineurinicos y otros similares pueden generar deterioro clínico y reconstitución inmune, no descrita con la suspensión de los corticoesteroides ${ }^{27}$. Por otro lado, se ha encontrado un efecto sinérgico entre los agentes antifúngicos y los inhibidores de la calcineurina ${ }^{28}$.

El pronóstico de estos pacientes es desfavorable. En un estudio en E. U. A. la mortalidad a los 90 días en pacientes sin VIH fue de $27 \%$, mucho mayor que la de los que presentan esta predisposición de base ${ }^{29}$, probablemente asociado a un reconocimiento tardío y la respuesta inmune disfuncional. En este grupo de pacientes, $C$. gattii tiende a causar lesiones granulomatosas pulmonares y cerebrales, con mayores secuelas neurológicas. La alteración del estado mental y la carga fúngica son factores relacionados al pronóstico; además, marcadores con pobre respuesta inflamatoria como un recuento bajo de linfocitos en LCR, ausencia de cefalea, cáncer hematológico o enfermedad hepática o renal crónica son marcadores de un peor pronóstico ${ }^{1}$.

Nuestros pacientes presentaron desenlaces fatales asociados a infecciones nosocomiales asociadas a la meningitis criptocóccica. Se destaca el espectro clínico y presentación distinta: uno con cefalea como síntoma predominante y el otro caso con un cuadro netamente confusional y con focalización neurológica.

\section{Resumen}

La criptococosis es una enfermedad fúngica de distribución global con una mayor incidencia en países en vía de desarrollo, donde constituye una de las principales causas de meningitis, asociado a una alta morbi-mortalidad. Afecta principalmente a pacientes con inmunosupresión (por infección por VIH, tratamiento con glucocorticoides, sometidos a trasplantes, cáncer, etc). Sin embargo, en los últimos años se ha presentado un incremento de casos en inmunocompetentes, siendo tanto o más grave que en inmunocomprometidos. Presentamos dos casos de meningitis criptocóccica. Un varón de 48 años, en quien se descartó una infección por VIH u otra causa de inmunosupresión y una mujer de 67 años con antecedentes de cirrosis hepática. Debe considerarse Cryptococcus spp. como posible agente causal de meningitis, tanto en pacientes inmunocomprometidos como inmunocompetentes. 


\section{Referencias bibliográficas}

1.- Pappas P G, Perfect J R, Cloud G A, Larsen R A, Pankey G A, Lancaster D J, et al. Cryptococcosis in human immunodeficiency virus-negative patients in the era of effective azole therapy. Clin Inf Dis 2001; 33: 690-9.

2.- Kiertiburanakul S, Wirojtananugoon S, Pracharktam R, Sungkanuparph S. Cryptococcosis in human immunodeficiency virus-negative patients. Int J Infect Dis 2006; 10: 72-8.

3.- Park B J, Wannemuehler K A, Marston B J, Govender N, Pappas P G, Chiller T M. Estimation of the current global burden of cryptococcal meningitis among persons living with HIV/AIDS. AIDS 2009; 23: 525-30. doi: 10.1097/QAD.0b013e328322ffac.

4.- Desalermos A, Kourkoumpetis T K, Mylonakis E. Update on the epidemiology and management of cryptococcal meningitis. Expert Opin Pharmacother 2012; 13: 783-9. doi: 10.1517/14656566.2012.658773.

5.- Rajasingham R, Rhein J, Klammer K, Musubire A, Nabeta H, Akampurira A, et al. Epidemiology of meningitis in an HIV-infected Ugandan cohort. American J Trop Med Hyg 2015; 92: 274-9. doi: 10.4269/ajtmh.14-0452.

6.- Zhu L P, Wu J Q, Xu B, Ou X T, Zhang Q Q, Weng X H. Cryptococcal meningitis in nonHIV-infected patients in a Chinese tertiary care hospital, 1997-2007. Med Mycol 2010; 48: 570-9. doi: 10.3109/13693780903437876.

7.- Pyrgos V, Seitz A E, Steiner C A, Prevots D R, Williamson P R. Epidemiology of cryptococcal meningitis in the US: 1997-2009. PloS One 2013; 8: e56269. doi: 10.1371/journal. pone.0056269.

8.- Phillips P, Galanis E, MacDougall L, Chong M Y, Balshaw R, Cook V J, et al. Longitudinal clinical findings and outcome among patients with Cryptococcus gattii infection in British Columbia. Clin Infect Dis 2015; 60: 1368-76. doi: 10.1093/cid/civ041.

9.- Currie B P, Casadevall A. Estimation of the prevalence of cryptococcal infection among patients infected with the human immunodeficiency virus in New York City. Clin Infect Dis 1994; 19: 1029-33.

10.- Pappas P G, Alexander B D, Andes D R, Hadley S, Kauffman C A, Freifeld A, et al. Invasive fungal infections among organ transplant recipients: results of the TransplantAssociated Infection Surveillance Network (TRANSNET). Clin Infect Dis 2010; 50: 110111. doi: 10.1086/651262.

11.- Williamson P R, Jarvis J N, Panackal A A, Fisher M C, Molloy S F, Loyse A, et al.
Cryptococcal meningitis: epidemiology, immunology, diagnosis and therapy. Nat Rev Neurol 2017; 13: 13-24. doi: 10.1038/ nrneurol.2016.167.

12.- Ahmad D S, Esmadi M, Steinmann W C. Idiopathic CD4 lymphocytopenia: spectrum of opportunistic infections, malignancies, and autoimmune diseases. Avicenna J Med 2013; 3 : 37-47. doi: 10.4103/2231-0770.114121.

13.- Lee Y C, Chew G T, Robinson B W. Pulmonary and meningeal cryptococcosis in pulmonary alveolar proteinosis. Aust N Z J Med 1999; 29 : 843-4.

14.- Saijo T, Chen J, Chen S C, Rosen L B, Yi J, Sorrell T C, et al. Anti-granulocyte-macrophage colony-stimulating factor autoantibodies are a risk factor for central nervous system infection by Cryptococcus gattii in otherwise immunocompetent patients. MBio 2014; 5: e00912-14. doi: 10.1128/mBio.00912-14.

15.- Sun H Y, Alexander B D, Huprikar S, Forrest G N, Bruno D, Lyon G M, et al. Predictors of immune reconstitution syndrome in organ transplant recipients with cryptococcosis: implications for the management of immunosuppression. Clin Infect Dis 2015; 60: 36-44. doi: 10.1093/cid/ciu711.

16.- Mitchell D H, Sorrell T C, Allworth A M, Heath C H, McGregor A R, Papanaoum $\mathrm{K}$, et al. Cryptococcal disease of the CNS in immunocompetent hosts: influence of cryptococcal variety on clinical manifestations and outcome. Clin Infect Dis 1995; 20: 611-6.

17.- Schoffelen T, Illnait-Zaragozi M T, Joosten L A, Netea M G, Boekhout T, Meis J F, et al. Cryptococcus gattii induces a cytokine pattern that is distinct from other cryptococcal species. PloS One 2013; 8: e55579. doi: 10.1371/ journal.pone.0055579.

18.- Hardison S E, Ravi S, Wozniak K L, Young M L, Olszewski M A, Wormley F L Jr. Pulmonary infection with an interferon-gamma-producing Cryptococcus neoformans strain results in classical macrophage activation and protection. Am J Path 2010; 176: 774-85. doi: 10.2353/ ajpath.2010.090634.

19.- Jitmuang A, Panackal A A, Williamson P R, Bennett J E, Dekker J P, Zelazny A M. Performance of the cryptococcal antigen lateral flow assay in non-HIV-related cryptococcosis. J Clin Microbiol 2016; 54: 460-3. doi: 10.1128/ JCM.02223-15.

20.- Nussbaum J C, Jackson A, Namarika D, Phulusa J, Kenala J, Kanyemba C, et al. Combination flucytosine and high-dose fluconazole compared with fluconazole monotherapy for the treatment of cryptococcal meningitis: a randomized trial in Malawi.
Clin Infect Dis 2010; 50: 338-44. doi: 10.1086/649861.

21.- van der Horst C M, Saag M S, Cloud G A, Hamill R J, Graybill J R, Sobel J D, et al. Treatment of cryptococcal meningitis associated with the acquired immunodeficiency syndrome. National Institute of Allergy and Infectious Diseases Mycoses Study Group and AIDS Clinical Trials Group. N England J Med 1997; 337: 15-21.

22.- Brouwer A E, Rajanuwong A, Chierakul W, Griffin G E, Larsen R A, White N J, et al. Combination antifungal therapies for HIV-associated cryptococcal meningitis: a randomised trial. Lancet 2004; 363: 1764-7.

23.- Day J N, Chau T T, Lalloo D G. Combination antifungal therapy for cryptococcal meningitis. N Engl J Med 2013; 368: 2522-3. doi: 10.1056/ NEJMc1305981.

24.- Perfect J R, Dismukes W E, Dromer F, Goldman D L, Graybill J R, Hamill R J, et al. Clinical practice guidelines for the management of cryptococcal disease: 2010 update by the Infectious Diseases Society of America. Clin Infect Dis 2010; 50: 291-322. doi: 10.1086/649858.

25.- Loyse A, Wilson D, Meintjes G, Jarvis J N, Bicanic T, Bishop L, et al. Comparison of the early fungicidal activity of high-dose fluconazole, voriconazole, and flucytosine as second-line drugs given in combination with amphotericin B for the treatment of HIVassociated cryptococcal meningitis. Clin Infect Dis 2012; 54: 121-8. doi: 10.1093/cid/cir745.

26.- Yao Z W, Lu X, Shen C, Lin D F. Comparison of flucytosine and fluconazole combined with amphotericin B for the treatment of HIV-associated cryptococcal meningitis: a systematic review and meta-analysis. Eur J Clin Microbiol Infect Dis 2014; 33: 1339-44. doi: 10.1007/s10096-014-2074-2.

27.- Singh N. How I treat cryptococcosis in organ transplant recipients. Transplantation 2012; 93: 17-21. doi: 10.1097/TP.0b013e318236cd1a.

28.- Kontoyiannis D P, Lewis R E, Alexander B D, Lortholary O, Dromer F, Gupta K L, et al. Calcineurin inhibitor agents interact synergistically with antifungal agents in vitro against Cryptococcus neoformans isolates: correlation with outcome in solid organ transplant recipients with cryptococcosis. Antimicrob Agents Chemother 2008; 52: 735-8.

29.- Brizendine K D, Baddley J W, Pappas P G. Predictors of mortality and differences in clinical features among patients with cryptococcosis according to immune status. PloS One 2013; 8: e60431. doi: 10.1371/ journal.pone.0060431. 